\title{
INFLUENCIA DEL MÉTODO TOMATIS $®$ EN LA REDUCCIÓN DE LA SINTOMATOLOGÍA EN UN NIÑO AUTISTA
}

\author{
'Michel Pralong, ${ }^{2}$ Elisa Espinosa, ${ }^{3}$ Eugenia Trigo
}

\author{
1Universidad Tecnológica Equinoccial, Quito - Ecuador, michelpralong@yahoo.fr \\ 2Ministerio de Educación / Universidad Tecnológica Equinoccial / Pontificia Universidad Católica del Ecuador, \\ Quito - Ecuador, asile_55@hotmail.com \\ ${ }^{3}$ Instituto Internacional del Saber Kon-traste, Viterbo - Colombia, etrigoa@gmail.com
}

Recepción / Received: 21, 07, 2014

Aceptación / Accepted: 17, 09, 2014

Publicado / Published: 12, 12, 2014

\section{Resumen}

Esta investigación cualitativa se enfocó en la observación y análisis de las reacciones de un niño de 8 años que presenta autismo, frente a la aplicación del método "Tomatis®”, que sirve para procesar mensajes acústicos mediante estimulación auditiva. Se utilizaron sesiones de escucha con temas musicales especializados y la aplicación del “Tooballo®”.Se realizaron observaciones in situ antes, durante y después de sesiones de estimulación Tomatis® y uso del Tooballo®. Asimismo, se aplicó al inicio, durante y al final el método de la SCAS (Spence Children's Anxiety Scale), el cual mide la ansiedad en niños y la evaluación (parental) del diagnóstico Vanderbilt de TDHA, que mide el nivel de hiperactividad. También se realizó un grupo focal con familiares de niños con autismo, con el tema: "Estrategias que utilizaron los padres para afrontar las crisis de sus hijos con autismo". Tras la implementación del método Tomatis® y el empleo del Tooballo® el sujeto de esta investigación logró un nivel notable de maduración evolutiva, disminuyeron los rasgos de ansiedad y progresó significativamente en el proceso de la lectura y escritura.

Palabras claves: Ansiedad, asperger, autismo, hiperactividad, método Tomatis®

\begin{abstract}
Pioneering Qualitative research project focused in the observation and analysis of a 8 years old autist kid reactions, with careful attention when submitted to Tomatis $₫$ method, a technique for processing acoustic messages through auditory stimulation by means of especially designed musical themes listening sessions and "Tooballo®" usage. In observations in situ before, during and after the listening of Tomatis ${ }^{\circledR}$ stimulation and Tooballo® usage were applied SCAS (Spence Children's Anxiety Scale), that measures a wide anxiety spectrum in kids, and Vanderbilt TDHA by means of parental diagnosis evaluation to measure hyperactivity level. A focal group with parents of autistic kids of CAPADD Foundation, regarding the following subject: "Strategic procedures implemented by parents in order to cope with their autistic children's crisis". Significant evolutionary maturing level was acquired, anxiety characteristics decreased and important progress was achieved in writing and reading skills.
\end{abstract}

Keywords: Anxiety, asperger, autism, hyperactivity, Tomatis $®$ method 


\section{INTRODUCCIÓN}

El niño objeto de este estudio de caso presentó un desarrollo evolutivo lento durante sus cuatro primeros años de vida. Caminó a los dos años y a los cuatro años podía expresarse con pocas palabras. A los tres años, a raíz del temor provocado por una intervención quirúrgica, se encerró más en su mundo. A los cuatro años fue diagnosticado con el síndrome de Asperger. El niño presentaba un alto grado de ansiedad, trastorno de déficit de atención, poca sociabilización, agresividad, conductas obsesivas, en particular la de hacer girar ruedas durante horas y retraso en la adquisición de los procesos de la lectoescritura. Tomaba medicina para disminuir su comportamiento agresivo e hiperactivo.

Conocido el método Tomatis® como una terapia aplicada a niños con autismo con cierto grado de efectividad (60\% de resultados positivos) (Gerritsen, 2009), el investigador decide utilizarlo con el propósito de comprobar si este método logra disminuir la sintomatología que presentaba el niño sujeto de estudio.

Las investigaciones realizadas con esta técnica de estimulación sonora dan cuenta de que los cambios en los niños con autismo van de pequeños a grandes progresos. Neysmith-Roy (2001), de la Universidad de Regina, Canadá, estudió el impacto de la intervención Tomatis ${ }^{\circledR}$ en seis niños con autismo severo. Ellos fueron valorados en la escala CARS (Child Autism Rating Scale), previamente y posteriormente al tratamiento. Tres de los chicos (A, C y D) demostraron cambios de comportamiento positivos al final del tratamiento. Uno de los chicos (A) ya no se consideró autista al final del tratamiento. Los dos otros chicos (C y D) mostraron solo síntomas benignos de autismo al final del tratamiento. Los tres últimos niños (B, E y F) mantuvieron sus niveles de puntuación de autismo severo. De los seis chicos, cinco de ellos mejoraron en las áreas prelingüísticas (adaptación al cambio, respuesta de la escucha, comunicación no verbal, respuesta emocional).

El trastorno del espectro autista consiste en una gama de trastornos complejos del neurodesarrollo caracterizado por tres tipos de disturbios principales (se habla de trilogía autista): la falta de comunicación (problemas de lenguaje, ecolalia), un centro de interés restringido con conductas estereotipadas repetitivas y, finalmente, la dificultad de establecer interacciones sociales (Emission sante 36.9, 2010). La angustia, ira, ansiedad, son algunos rasgos que una gran parte de niños autistas padecen. Las personas que sufren del síndrome de Asperger tienen un coeficiente intelectual medio o superior a lo normal y habitualmente no tienen normalmente un retraso en la adquisición de habilidades lingüísticas.

La Dra. M. Delfos (2005, 2011), psicologa clínica y psicoterapeuta belga, presenta una visión sobre al autismo diferente a lo habitual. Para ella, las personas con autismo, si no tomamos en cuenta la cuarta parte de los autistas que poseen una discapacidad mental, no presentan una deficiencia. Según su punto de vista, se trata de un perfil desarmónico, un desbalance del desarrollo, de un cerebro demasiado masculino, es decir, de un cerebro más desarrollado en el hemisferio derecho, más físico (interés sobre las cosas - ejemplos: ruidos, reloj, etc.) y no lo suficientemente desarrollado en el hemisferio izquierdo que, según estudios neurocientíficos, alberga más la auto consciencia y la parte social; por este perfil, los niños con autismo tendrán mayor problema para relacionarse.

Las preguntas que se formularon en el presente estudio fueron las siguientes:

¿Podrá el método Tomatis ${ }^{\circledR}$ reducir los trastornos (angustias, estrés, ira) que padece un niño autista? Caso: Nicolás.

¿Cuáles son las conductas observables que manifiesta Nicolás, diagnosticado como un niño autista-Asperger? ¿Cuáles son las consecuencias para Nicolás?

¿Qué se puede hacer para reducir las crisis (angustias, estrés, ira, etc.)?

¿El método Tomatis $®$ podría disminuir las crisis que tienen la mayoría de los niños autistas, y en este caso, las de Nicolás?

¿El método Tomatis ${ }^{\circledR}$ podría mejorar los procesos de lectoescritura en Nicolás?

¿Cuáles son las estrategias que utilizan los padres de los niños autistas para afrontar las crisis?

Según la Dra. Delfos, el desarrollo humano va acorde con algunas fases, que no se pueden saltar. Para ayudar lo más armónicamente posible al niño es necesario conocer la edad de desarrollo social, así como tener en cuenta su edad cronológica. Ejemplo: si a un niño le quitamos un juguete a los seis meses, este no reaccionará, pero si se lo quitamos a un niño que tiene 2 años, empezará a gritar. Esto es lo que se conoce como desarrollo "normal" del niño. Entonces, lo fundamental es descubrir en qué etapa del desarrollo se encuentra el niño (desarrollo motor, psíquico, emocional,...) y relacionarse con él en función de la edad que tenga en este momento. Por tanto es oportuno hacernos estas dos preguntas:

- ¿La conducta de este niño es "normal" por su edad? - ¿Qué podemos hacer, educadores y padres para mejorar esta conducta?

El enfoque de esta psicoterapeuta se basa en terapias

| 14 | REVISTA EÍDOS, diciembre 2014 
lúdicas (utiliza juegos como el de las escondidas, el caballito y el monstruo), acordes a las edades de desarrollo del niño, a través de los cuales resulta posible el desarrollo de muchas habilidades, al tiempo que posibilita la detección de lo que necesita el niño para crecer.

Esta psicóloga clínica, investigadora y autora de varios libros, entre ellos Un mundo extraño, publicado en 2004, entiende de esta manera el autismo. Sus trabajos recién se están tomando en cuenta en el mundo científico, tal y como se hace en este estudio.

El Dr. Alfred Tomatis (1920 - 2001), fue el creador del método Tomatis®. Se trata de una técnica de estimulación sensorial sonora que se basa en un dispositivo diseñado para hacer trabajar los músculos del oído con el objetivo de restaurar sus capacidades completas para transmitir y, sobre todo, procesar el mensaje acústico gracias a un sistema de dos canales de transmisiones sonoras (que amplifican la intensidad y modifican el timbre). Este dispositivo envía sonidos capaces de contraer y relajar los músculos del oído, el músculo del estribo y el del martillo (en el oído medio). Por otra parte, aunque el sonido se transmite por el aire, este primero es transmitido, mediante la vibración del hueso, por conducción ósea, gracias a unos audífonos especialmente equipados.

Esta vibración permite al oído anticipar el mensaje que va a recibir a través del tímpano y transmitirlo más eficientemente al cerebro. Tras varias y repetidas sesiones, el oído y el cerebro ya no necesitan la máquina para que la cualidad de la escucha se desarrolle y se afiance (Tomatis, 2011) (véase Figura $\left.N^{0} 1\right)$

El Método Tomatis ${ }^{\circledR}$ consiste en un método que pretende reeducar la forma de escuchar, mejorar el aprendizaje, las habilidades del lenguaje, la atención, la concentración, la comunicación, la creatividad, el comportamiento social, así como proporcionar una mayor actividad cerebral y dinamizar el cuerpo.

El Método Tomatis $®$ cuenta con el aparato Solisten $®$, (Figura No 2) un poco más grande que un Mp3, que contiene
8 programas musicales y sus características técnicas, la pantalla es táctil y es de muy sencillo uso; viene con su audífono especial de conducción ósea y aérea, a través de los cuales envían al oído sonidos de los programas musicales ya referidos.

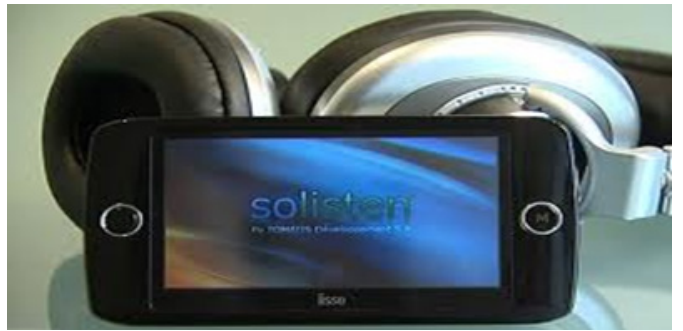

Figura $\mathrm{N}^{\circ}$ 2. El Solisten®

Fuente: Tomatis Development

Los programas que tiene son:

- El "Básico", con el cual se empieza siempre, cualquiera sea la dificultad a enfrentar. Proporciona una gran cantidad de energía, gracias a la música de Mozart (trabaja sobre todas las frecuencias de $125 \mathrm{~Hz}$ a $16000 \mathrm{~Hz}$ ). Este programa no tiene filtros para acostumbrar a la persona a todas las frecuencias. Se utiliza en pacientes que presentan problemas importantes para procesar la información, y para problemas motores (planificación, coordinación, ritmo, postura). Además de la música de Mozart hay también cantos gregorianos que ayudan a regular el sistema neurovegetativo, parte del sistema nervioso que regula las funciones de nutrición, desarrollo y reproducción, y que trabaja mucho en la esfera vestibular.

- El "Emocional", que está compuesto por tres programas derivados: E1A, E1B y E2. Los programas E1A y E2 incluyen música densificada que tiene la función de estimular el córtex prefrontal, el cual, además, de ser un regulador emocional, es responsable de llevar a cabo los procesos ejecutivos. El E1B ha sido creado para personas que no pueden ser sometidas a la música densificada por presentar problemas de epilepsia o vértigos. Los programas emocionales trabajan a nivel vestibular y se utilizan para los problemas de ansiedad, depresión, estrés, problemas de irritabilidad y problemas psicomotores.

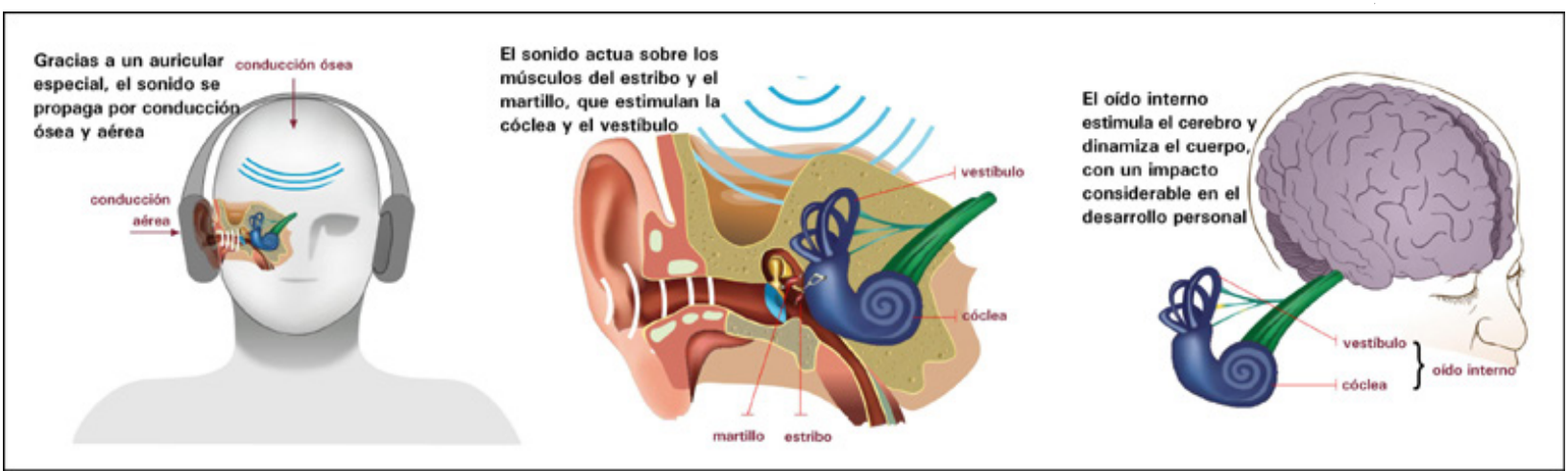

Figura $N^{\circ} 1$. Funcionamiento del Método Tomatis.

Fuente: Tomatis Development 
- El "Lenguaje" que se compone de dos programas, el L1 y el L2. Se aconseja para casos de falta de energía, un retraso en el lenguaje, dificultades para leer, cuando hay dificultades en la percepción del habla y en el análisis de los sonidos y para el aprendizaje de idiomas.

- El programa "MA" (Memoria y Atención) que se divide en dos, el M1 y el M2. Este programa, además de ayudar con los problemas de atención, estimula de manera importante y crea mucha energía cortical que actúa de cierta manera como la Ritalina, medicamento que se administra a menudo a los niños hiperactivos. Asimismo, es aconsejable cuando hay problemas de memoria, dificultades de planificación y organización, y de fluidez verbal.

También se trabaja con el Toobaloo®, (Figura No 3) aparato especial de material plástico que permite al portador escucharse hablar sin que se interpongan ruidos mientras habla y que forma parte de la reeducación del oído (amplifica las frecuencias lingüísticas).

Siempre se trabaja de una manera global, y no en una forma específica. Cada sesión dura 15 días y el tiempo

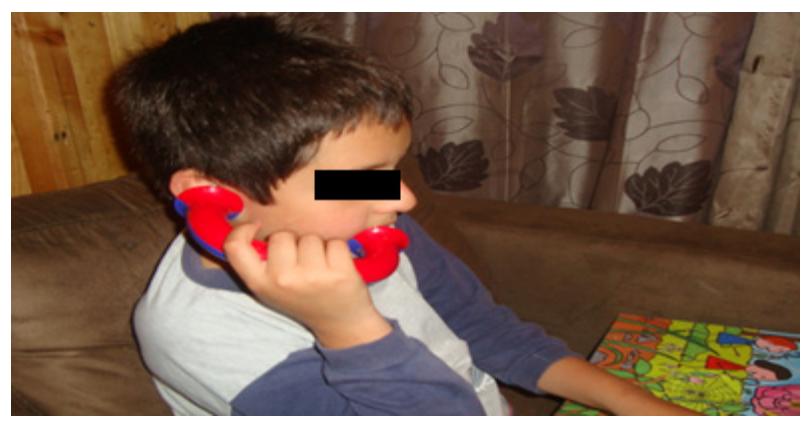

Figura $N^{\circ} 3$. El niño en estudio trabajando con el Toobaloo® Fuente: Michel Pralong, autor principal

adecuado de escucha es de 2 horas diarias (4 músicas de 30 minutos cada una). Posteriormente se realiza una pausa de un mes y se hace una evaluación para analizar cómo se sintió el paciente durante el proceso de escucha. Durante este periodo deberá leer o hablar sobre un tema con la ayuda del Toobaloo®. Una vez acabado el trabajo con el Toobaloo®, se hace una nueva evaluación del paciente. La elección del programa de escucha siguiente dependerá de los resultados adquiridos y así, sucesivamente, las pausas serán siempre más largas (Tomatis, 2011).

Según la experiencia de varios practicantes Tomatis, en muchos casos, el tratamiento se puede detener después de tres sesiones, pero un tratamiento puede durar varios años, como en el caso del autismo y también puede no ser efectivo.

Se debe recalcar que no se puede aplicar cualquier programa a cualquier persona, pues siempre se debe realizar una estricta evaluación, antes y después de las escuchas y de la utilización del aparato Toobaloo®. Es por esta razón que, antes de empezar una programación con un nuevo paciente, es necesario visualizar si se requiere, para ayudar a resolver el problema, de frecuencias graves, medianas o agudas dentro de la línea que va desde 0 a 16000 Hertz y, así mismo, dentro de qué zona se necesita trabajar, pues mientras más se empleen filtros, menos báscula existirá y más energía habrá. La báscula, denominada "báscula electrónica con efecto Tomatis ${ }^{\circledR}$ ", es un sistema que crea un contraste perceptivo, gracias a la alternancia de un sonido en timbre y en intensidad. Por un reflejo natural, los músculos del oído se activan bajo el efecto del contraste y estimulan el sistema vestibular, nervioso y cerebral. El "efecto Tomatis@" permite entonces al cerebro que mejore la interpretación del mensaje sensorial, de forma natural, y así aprende a escuchar mejor. Hablando de escuchar, el Dr. Tomatis $₫$ fue, gracias a sus investigaciones, el primero en hacer la diferencia entre oír y escuchar y subrayó los estrechos lazos que unen las diferentes partes del oído con el cerebro y el cuerpo. Según Tomatis, "la escucha representa para la audición lo que la puntería representa para la vista".

\section{METODOLOGÍA}

La presente investigación se basa en un estudio de caso descriptivo de tipo cualitativo, flexible, que emplea la observación de los comportamientos del niño sujeto de estudio durante y después de la aplicación del Método Tomatis ${ }^{2}$.

Se aplicó al sujeto 4 sesiones de escuchas, en un espacio de 9 meses con intervalos respectivamente de 1 mes, 1 mes y medio, 2 meses y 2 meses y medio durante los cuales el niño trabajó con el aparato Toobaloo $®$, entre 5 a 10 minutos diarios. El investigador recibió asesoramiento de la Dra. Valérie Gas, especialista del Método Tomatis $₫$, para pedirle consejos a la hora de escoger entre los programas. Durante los nueve meses, el niño se sometió 2 veces al programa Básico porque se manifestaron reacciones positivas en la primera sesión, lo que fue confirmado durante la segunda sesión. Luego, se le aplicó el programa "Emocional", principalmente para disminuir su nivel de ansiedad y estrés. El tercer programa escogido fue el de Lenguaje, que el niño escuchó durante las 30 horas que duró su aplicación. El objetivo era, pues, ayudarle a superar sus problemas a nivel escolar. Finalmente, el programa "MA" (Memoria y Atención) fue escogido para que actúe como tranquilizante de sus crisis de hiperactividad.

Antes, durante y después de la aplicación del Método Tomatis ${ }^{\circ}$ se aplicó el cuestionario de conductas observables de la escala de ansiedad de Spence para medir su nivel de angustia y estrés(Herdandez-Guzmán ET $A L, 2010)$.

La SCAS (Spence Children's Anxiety Scale) fue construida

| 16 | REVISTA É́DOS, diciembre 2014 
por Spence en 1997, en Australia. Es un instrumento de autoinforme al que responden los niños o sus padres, el cual consta de 44 ítems. Se desarrolló con la intención de investigar una gama amplia de síntomas de ansiedad en niños de la población general. Los estudios realizados por su autora han corroborado la coincidencia de su estructura factorial de seis trastornos de ansiedad. Seis ítems miden ansiedad de separación, seis de trastornos obsesivocompulsivos, seis de ataques de pánicos y agorafobias, seis de ansiedades generalizadas, seis de fobias sociales y, finalmente, cinco de miedo al daño físico. Se califica en una escala de cuatro opciones que fluctúan entre 0 , para nunca, y 3, para siempre (Herdandez-Guzmán ET AL, 2010).

De igual forma se llenó una evaluación (parental) del diagnóstico Vanderbilt de TDHA (NIOCHQ, 2010) para medir su nivel de hiperactividad. La evaluación está dividida en 47 observaciones en la cual hay 4 divisiones por respuestas, que representan los siguientes valores: $0=$ Nunca, $1=A$ veces, 2 = A Menudo y $3=$ Muy a menudo. Existen también divisiones en subtipos. Nueve observaciones corresponden a la falta de atención predominante, otras nueve miden la hiperactividad/impulsividad predominante. Un trastorno de oposición con provocación es otro subtipo que contiene 8 observaciones. Hay 14 conductas observables para el trastorno del comportamiento y 7 para identificar la ansiedad o la depresión, es decir, en total 47.

Se llenó igualmente el documento "Dificultades del aprendizaje de A a Z", que es una lista de dificultades del aprendizaje, escrito por Smith y Strick (1997), que reflejan si un niño tiene dificultades graves, medianas, leves o no, en su proceso de aprendizaje. Al niño sujeto de estudio se le observó el proceso de lectoescritura.

Se trabajó también con un grupo focal de 5 padres de familia de niños autistas, de la fundación CAPADD, en Quito, del especialista en autismo, el Sr. Ir. Norbert Groot, para conocer las "estrategias que utilizan los padres para afrontar las crisis de sus hijos con autismo". Se realizó la observación constante de las reacciones del niño frente a la aplicación del Método Tomatis ${ }$. Se utilizó el método comparativo de forma constante (análisis continuo de datos).

\section{RESULTADOS}

Durante los nueve meses que duró la aplicación del Método Tomatis $®$ se observó en el sujeto la disminución paulatina de sus niveles de ansiedad, pero no hubo cambios significativos en la atención e hiperactividad. En la parte académica hubo adelantos en el proceso de lectoescritura (en una semana logró asir el lápiz haciendo la pinza, lo que habían tratado que haga - sus profesores y padresdurante dos años). También hay cambios conductuales significativos como: fijar más la mirada, es más sensible y puede exteriorizar sus intereses, imita a los demás, tiene sentido del humor, comportamientos que no se hallan, en general, presentes en los niños con autismo. Se siente un poco más cómodo al presentarse frente al público. Está, también, más abierto a cambios impredecibles y busca ya el contacto con sus pares. Hace reflexiones, utiliza un léxico, a veces, de un niño mucho mayor que él.

Se le suprimió el medicamento que le administraban para reducir su ansiedad (Risperidona), sin haberse afectado su comportamiento. Se observó también que exterioriza más sus intereses, como cocinar, escuchar música, hacer trabajos con herramientas eléctricas y dejó atrás sus conductas obsesivas de quedarse horas girando cosas o conectar aparatos eléctricos.

Hubo también progresos enormes de lógica operativa: se ha podido notar eso observándole jugar con un juego en video: actúa con mucho juicio. Sin embargo, los gestos estereotipados (aletear, girar sobre sí mismo) no disminuyeron en el sujeto, tampoco las obsesiones poco frecuentes, aunque fuertes, que provenían de frustraciones al no poder lograr lo que estaba predispuesto a hacer o a conseguir. Todavía no sabe esperar su turno, no acepta que alguien sea mejor que él, sobre todo cuando es dentro del marco de un espíritu "competitivo".

Tabla No1.

Conductas observables del niño en estudio. Fuente: Michel Pralong, autor principal

A continuación, en la Tabla No 1, una síntesis de las conductas observables del niño en estudio:

Tabla № 1. Conductas observables del niño en estudio. Fuente: Michel Pralong, autor principal

\begin{tabular}{|c|c|c|c|}
\hline Sintomatología & $\begin{array}{c}\text { Al inicio: } \\
\text { abril de } 2012\end{array}$ & $\begin{array}{c}\text { En la mitad: } \\
\text { septiembre de } \\
2012\end{array}$ & $\begin{array}{c}\text { Al final: } \\
\text { enero de } 2013\end{array}$ \\
\hline $\begin{array}{l}\text { Ansiedad/ } \\
\text { Angustia }\end{array}$ & $\begin{array}{l}\text { Gran nivel de } \\
\text { ansiedad (42) }\end{array}$ & $\begin{array}{c}\text { El nivel de } \\
\text { ansiedad baja } \\
\text { enormemente } \\
\text { en relación a } \\
\text { abril de } 2012 \\
\text { (28) }\end{array}$ & $\begin{array}{c}\text { El nivel de } \\
\text { ansiedad } \\
\text { baja todavía } \\
\text { un poco más } \\
\text { en relación a } \\
\text { septiembre de } \\
2013(22)\end{array}$ \\
\hline Hiperactividad & $\begin{array}{l}\text { Nivel elevado de } \\
\text { hiperactividad } \\
(60)\end{array}$ & $\begin{array}{c}\text { El nivel de } \\
\text { hiperactividad } \\
\text { baja un poco } \\
\text { en relación a } \\
\text { abril de } 2012 \\
\text { (56) }\end{array}$ & $\begin{array}{c}\text { El nivel de } \\
\text { hiperactividad } \\
\text { baja } \\
\text { sensiblemente } \\
\text { en relación a } \\
\text { septiembre de } \\
2012 \text { (51) }\end{array}$ \\
\hline
\end{tabular}

\section{DISCUSIÓN / CONCLUSIONES}

A primera vista, estos datos indican que el Método Tomatis $₫$ redujo la sintomatología del niño en estudio.

Se produjeron cambios significativos en los niveles de 
ansiedad, en los comportamientos sociales y en los aprendizajes escolares que fueron medidos a través de las escalas de ansiedad de Spence y la escala de Hiperactividad de Vandervilth. Los programas musicales del Método Tomatis $®$ aplicados durante los nueve meses estimularon los músculos del oído, ayudando a procesar el mensaje acústico que restauró algunas capacidades como el mejorar su crisis de ansiedad, disminuir la hiperactividad aunque no en el grado que se hubiera esperado y mejorar sus aprendizajes escolares.

Quizá estos logros coincidieron con la madurez evolutiva del niño, o tal vez apoyaron concomitantemente al proceso de desarrollo individual según lo que plantea Martina Delfos (2005, 2011). De cualquier modo el desarrollo desarmónico del niño de estudio fue estimulado y pudo mejorar su desenvolvimiento social, afectivo y escolar.

Las impresiones del investigador/padre señalan que durante los nueve meses que duró la investigación, fueron como una gestación, y como tal no fue una "aventura" totalmente apacible. El hecho de ser el investigador, y padre a la vez, del único estudio de caso, fue a veces difícil, porque tuvo que evaluar al niño, analizar los resultados de la manera más neutra, perspicaz, precisa y objetiva que fuera posible. Pasó por todos los estados: preocupación, entusiasmo, abatimiento, interés, alegría y ha tenido que cuestionarse en repetidas ocasiones para cambiar su actitud, armarse de paciencia, y volver a armarse de paciencia y sacar fuerzas de donde fuese, a fin de llevar a cabo de la mejor manera y forma posibles esta investigación. Una persona con mucha sabiduría hizo al autor de esta investigación la reflexión siguiente, lo que le dio bastante para elucubrar: "Mientras más tratas que el niño entre en tu mundo, más se alejará; mientras más trates de entrar en el mundo de él, más se acercará". El "investigador/padre" piensa que alcanzó a veces a traspasar los muros de esa fortaleza, antes inexpugnable, y entender mejor al príncipe que vive en ella, y proporcionarle algo de las luces que requería para iniciar un camino en el mundo que fuera de ella se ubica.

De todas formas, se reacciona de manera compleja e interrelacionada ante cada estímulo que se recibe, o situación que se vive, etc. Eso es lo que constituye lo humano. De ahí que la investigación cualitativa que enfrenta problemas humanos, como el caso del niño de estudio se convierta en pertinazmente humana. No medimos, no comparamos, no damos por cierto como la vida misma enseña.

\section{REFERENCIAS BIBLIOGRÁFICAS}

Delfos, M. (2011). Reunión/taller con la Dra. Martine Delfos y Norbert Groot, el 21 de noviembre de 2011 en el centro CAPADD, en Quito.
Delfos, Marine (2005) A Strange World - Autism, Asperger's Syndrome and PDD-NOS: A Guide for Parents, Partners, Professional Careers, and People with ASDs. Londres \& Filadelfia: Jessica Kingsley Publishers.

Emission santé 36.9 (2010)« Au cœur du cerveau autiste " de la RTS (Radio Télévision Suisse Romande) . Recuperado de http://www.rts.ch/emissions/369/2469825-au-coeur-du-cerveau-autiste.html

Adicionalmente, al respecto ver entre los trabajos del Científico Jean-Didier Vincent, miembro de la Academia de Ciencias de Francia y del CNRS, tales como: (2009). Viaje Extraordinario al Centro del Cerebro. Barcelona: Ed. Anagrama.

Gerritsen, J, 2009. Revisión de las Investigaciones hechas sobre Estimulación Auditiva Tomatis $®)$. Informe obtenido por el grupo Tomatis el 15 de agosto de 2012.

Herdandez-Guzmán, L Bermudez-Ornelas, G., Spence, S.H., Gonzalez, M., Martínez Guerrero, J., Aguilar, J., y J. Gallegos. (2010). Versión en español de la Escala de Ansiedad para Niños de Spence (SCAS). Revista Latinoamericana de Psicología 42(1), 13-24.

Neysmith-Roy. (2001). Revisión de las investigaciones hechas sobre Estimulación Auditiva Tomatis®. Informe obtenido por el grupo Tomatis el 15 de agosto de 2012

NICHQ. (2010). NICHQ Vanderbilt Assesment Scales. Bostos: $\mathrm{NICHQ}$.

Smith, C. y Strick, L. (1997). Learning Disabilities: A to Z. New York: The FreePress/Simon \& Schuster, Inc.

Tomatis. (2011). Material del Programa de formación Tomatis, practicante nivel 1. Panamá enero de 2012.

| 18 | REVISTA É́DOS, diciembre 2014 